\title{
I nstant stent-accentuated three-dimensional optical coherence tomography of a true bifurcation lesion treated with culotte stenting
}

\author{
Fumiaki Nakao \\ Department of Cardiology, Yamaguchi Grand Medical Center, Yamaguchi, Japan \\ Correspondence: Fumiaki Nakao. Address: Department of Cardiology, Yamaguchi Grand Medical Center, 77 Ohsaki, \\ Hofu, Yamaguchi 747-8511, Japan. E-mail: nakao-ymghp@umin.ac.jp \\ Received: September 30, 2014 Accepted: October 26, 2014 \\ Online Published: October 30, 2014 \\ DOI : $10.5430 /$ jbgc.v4n4p26 \\ URL: http://dx.doi.org/10.5430/jbgc.v4n4p26
}

\section{Abstract}

A 65-year-old woman was performed percutaneous coronary intervention for a bifurcation lesion of the left anterior descending artery (LAD) and the first diagonal branch (Dg). After pre-dilatation, the proximal LAD-Dg was stented with the platinum-chromium elevolimus-eluting stent (PtCrEES). After rewiring to LAD, the instant stent-accentuated 3-dimensional optical coherence tomography (iSA3D-OCT) was reconstructed from 2-dimensional OCT (2D-OCT) by Image J with self-made macro-program. Classification of overhanging struts in front of the side branch (SB) ostium was the connecting to carina type, and the recrossed wire was confirmed to pass through the large distal cell. After kissing balloon dilatation (KBD), the mid-distal LAD and the proximal-mid LAD were stented with PtCrEESs. After rewiring to Dg, classification of overhanging struts in front of the SB ostium was the free carina type, and the recrossed wire was confirmed to pass through the distal cell, according to iSA3D-OCT. After final KBD, final iSA3D-OCT showed no floating struts.

\section{Key words}

True bifurcation, Percutaneous coronary intervention, Culotte stenting, 3D-OCT

\section{Introduction}

We developed the instant stent-accentuated 3-dimensional optical coherence tomography (iSA3D-OCT) to confirm the relation between stent struts and the jailed side branch (SB), the rewiring cell during a bifurcation stenting ${ }^{[1,2]}$. Stent struts in 2-dimensional OCT (2D-OCT) are represented as high-intensity line segments or spots in low-intensity background. Using freeware ImageJ v1.47 (National Institutes of Health) with self-made macro-program, a vessel image is created from an original image by the mean filter followed by the minimum filter. A strut image is created by subtracting a vessel image from an original image, and accentuated. iSA3D-OCT is reconstructed from iSA2D-OCT created by adding a vessel image to a strut image. It takes about 30 seconds to create iSA3D-OCT from 2D-OCT by off-line computer. It usually takes about 2 min (meanwhile, the operator considers 2D-OCT) from an export of the image data to read the reconstructions and get sufficient conclusions. 


\section{Case presentation}

A 65-year-old woman with ST-elevated inferior myocardial infarction had been admitted after emergent percutaneous coronary intervention (PCI) for mid RCA, and was performed elective PCI for a bifurcation lesion of the left anterior descending artery (LAD) and the first diagonal branch (Dg) (Medina type 1,1,1) (see Figure 1). After pre-dilatation, the proximal LAD-Dg was stented with $2.25 \mathrm{~mm} \times 24 \mathrm{~mm}$ platinum-chromium elevolimus-eluting stent (PtCrEES) (Promus Premier, Boston Scientific Co.) (see Figure 2). After rewiring to LAD, X-ray fluoroscopy showed distal rewiring (see Figure 3). iSA3D-OCT was reconstructed from 2D-OCT (Dragonfly JP; St. Jude Medical). Classification of overhanging struts in front of the SB ostium was the connecting to carina type, and the recrossed wire was confirmed to pass through the large distal cell ${ }^{[3]}$ (see Figure 4). It was predicted the incomplete stent apposition (ISA) after final kissing balloon dilatation (KBD).
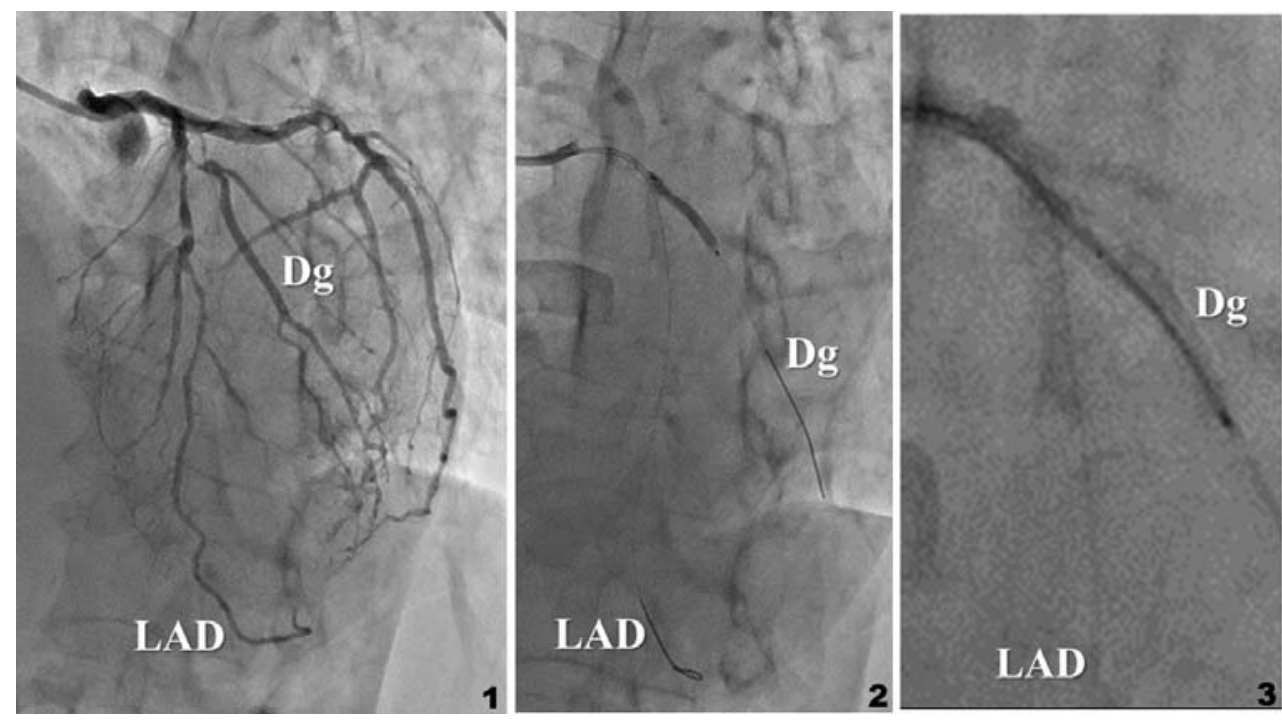

Figure 1. Baseline CAG showed true bifurcation lesion of LAD and Dg

Figure 2. Proximal LAD-Dg was stented

Figure 3. Rewiring to LAD

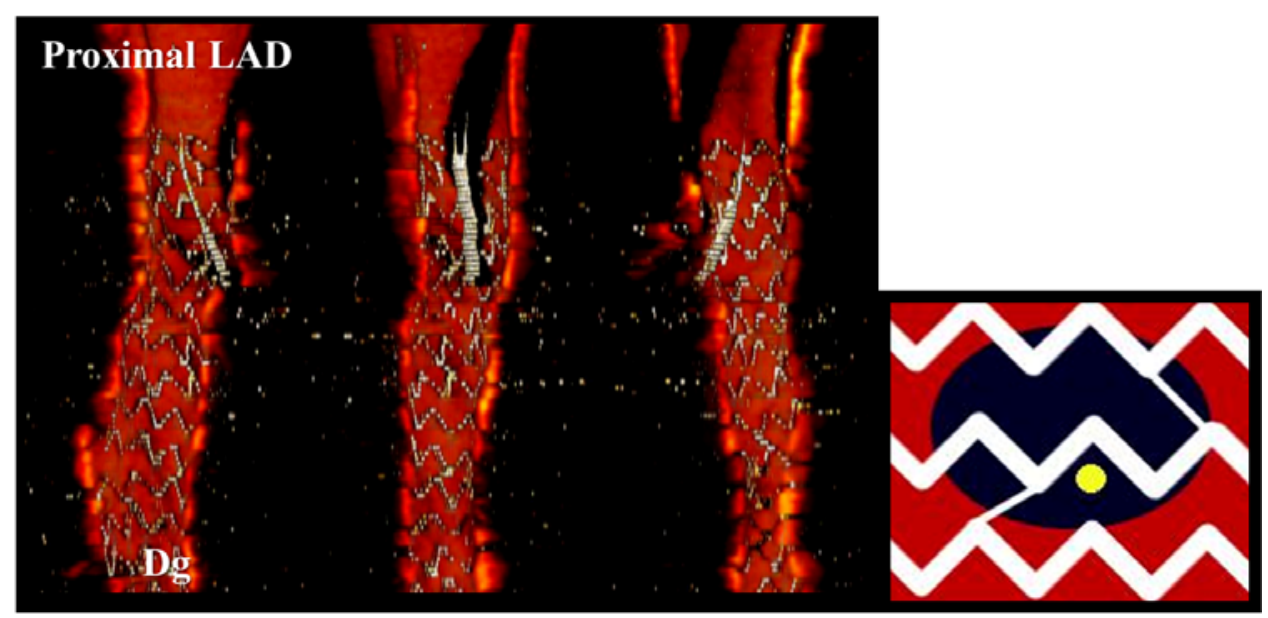

Figure 4. iSA3D-OCT after rewiring to LAD (rotational longitudinal cut-away view) showed connecting to carina type and large distal cell rewiring. Yellow dot in schema of jailing type on side branch ostium indicates rewiring cell.

After KBD, the mid-distal LAD was stented with $2.25 \mathrm{~mm} \times 20 \mathrm{~mm}$ PtCrEES and the proximal-mid LAD was stented with $3.0 \mathrm{~mm} \times 38 \mathrm{~mm}$ PtCrEES (see Figure 5). After rewiring to Dg, X-ray fluoroscopy showed mid-proximal rewiring (see Published by Sciedu Press 
Figure 6). According to iSA3D-OCT, classification of overhanging struts in front of the SB ostium was the free carina type, and the recrossed wire was confirmed to pass through the distal cell ${ }^{[3]}$ (see Figure 7). It was predicted ISA after final KBD and re-rewiring was recommended by a 2D modality, however, by iSA3D-OCT, it was predicted good apposition after final KBD. Final KBD was performed by simultaneously inflating $3.0 \mathrm{~mm} \times 12 \mathrm{~mm}$ and $2.0 \mathrm{~mm} \times 15 \mathrm{~mm}$ balloons (see Figure 8, 9). Final iSA3D-OCT showed good stent apposition and no floating struts (see Figure 10, 11).

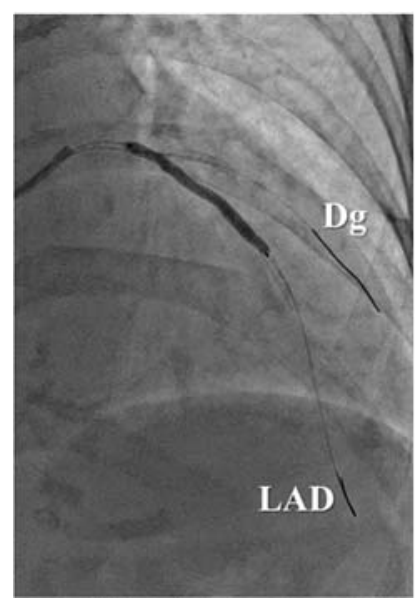

Figure 5. Proximal-mid LAD was stented

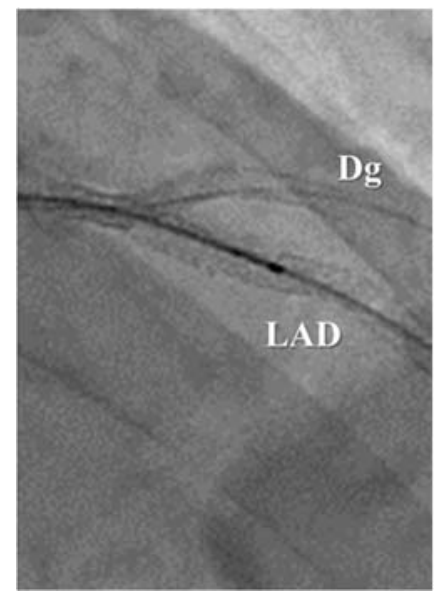

Figure 6. Rewiring to Dg

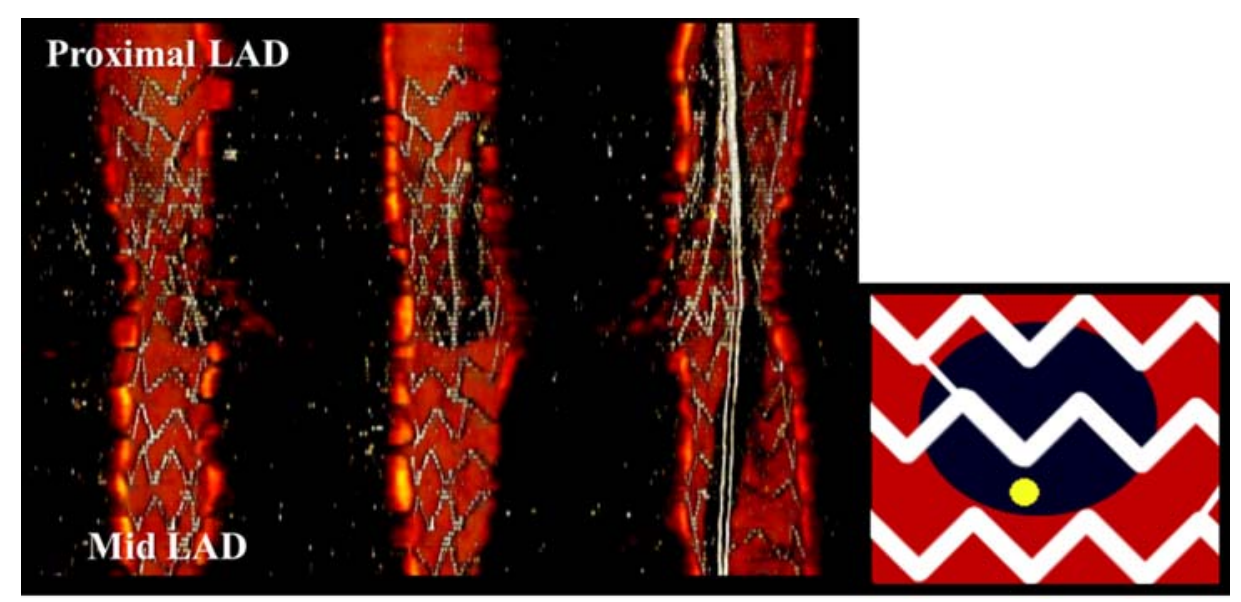

Figure 7. iSA3D-OCT after rewiring to Dg (rotational longitudinal cut-away view) showed free carina type and distal cell rewiring. Yellow dot in schema of jailing type on side branch ostium indicates rewiring cell.

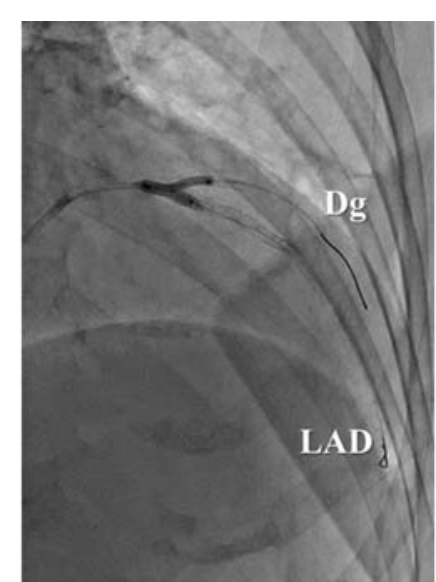

Figure 8. Final kissing balloon dilatation was performed

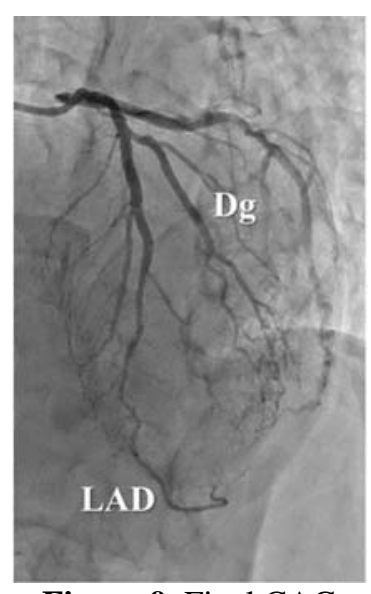

Figure 9. Final CAG 


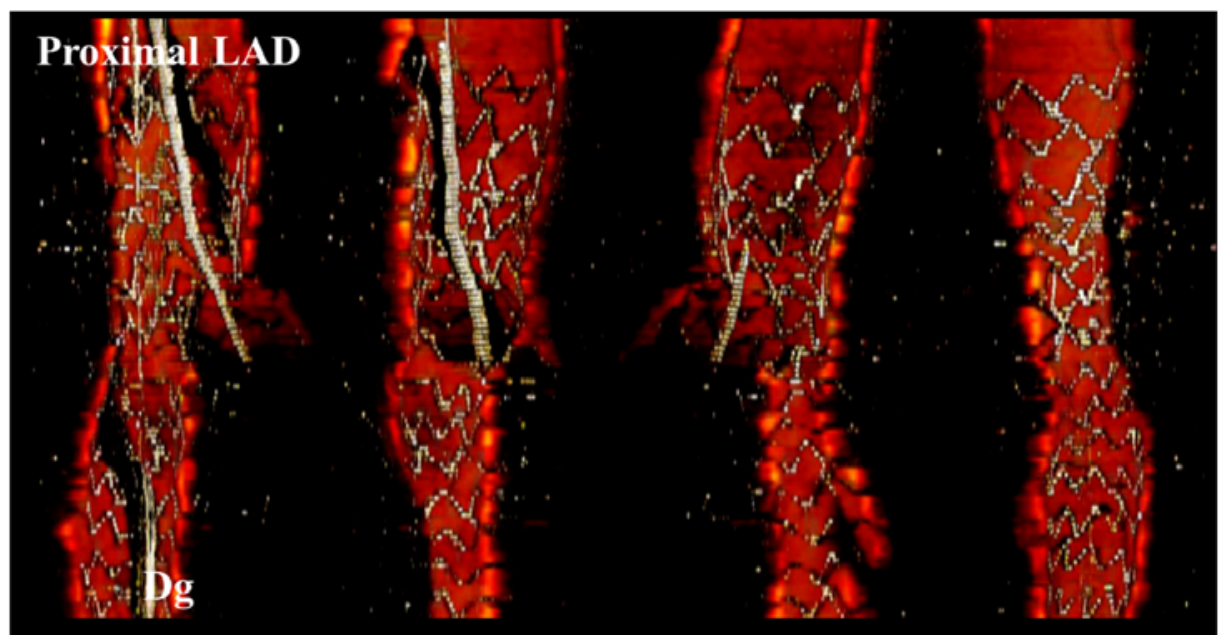

Figure 10. Final iSA3D-OCT (proximal LAD-Dg, rotational longitudinal cut-away view) showed no floating struts.

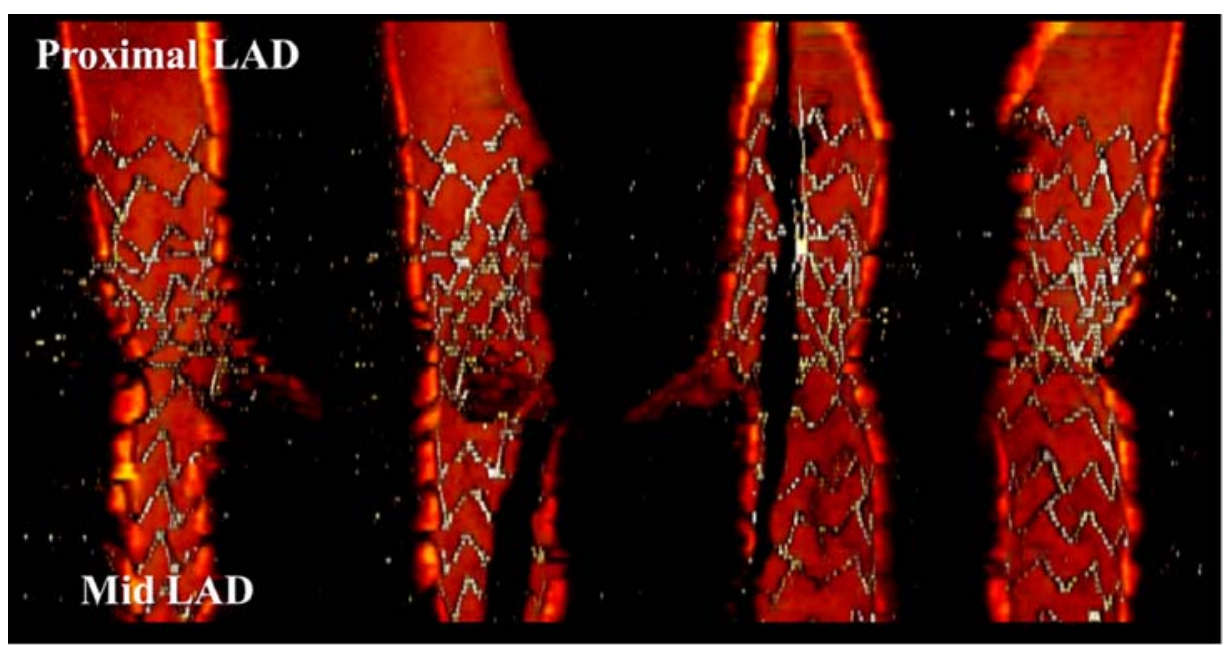

Figure 11. Final iSA3D-OCT (proximal-mid LAD, rotational longitudinal cut-away view) showed no floating struts.

\section{Discussion}

Generally the distal rewiring on a SB ostium has been recommended ${ }^{[4]}$. The ISA is a factor of poor prognosis ${ }^{[5]}$ and the proximal rewiring is a cause of ISA ${ }^{[4]}$. Okamura $\mathrm{T}$, et al. reported using 3D-OCT that, if both the favorable strut configuration and the distal rewiring, ISA after SB dilatation was reduced ${ }^{[3]}$. The distal rewiring confirmed by 2D modalities may be insufficient to reduce ISA. A confirmation of a rewiring cell by iSA3D-OCT during a bifurcation stenting is important to find such cases.

PtCrEES has the Element structure. By the unique structure, PtCrEES is very flexible, but more easily compressed and elongated than other stents ${ }^{[6]}$. The longitudinal weakness may serve as an advantage conversely. Because of the longitudinal weakness of PtCrEES, the round hole may be opened on the SB ostium by KBD, even if the connecting to carina type. ISA of first stent may influence to the apposition of the second stent in case of the culotte stenting, and therefore PtCrEES is useful for bifurcation stenting.

Using iSA3D-OCT, a detailed process during a bifurcation PCI can be confirmed in very short waiting time. It is expected to improve the outcome of a complicated bifurcation PCI by iSA3D-OCT. 


\section{Acknowledgement}

The author appreciates the members of the cardiovascular catheterization laboratory of Yamaguchi Grand Medical Center, and thanks to Tooru Ueda, M.D., Takamasa Oda, M.D., Masashi Kanemoto, M.D., Ph.D., Yasuhiro Ikeda, M.D., Ph.D., Takashi Fujii, M.D., Ph.D., of Department of Cardiology, Ymaguchi Grand Medical Center, for their supports, and Takayuki Okamura, M.D., Ph.D., of the Division of Cardiology, Department of Medicine and Clinical Science, Yamaguchi University Graduate School of Medicine, for his helpful advices.

\section{References}

[1] Nakao F, Ueda T, Nishimura S, Uchinoumi H, Kanemoto M, Tanaka N, et al. Novel and quick coronary image analysis by instant stent-accentuated three-dimensional optical coherence tomography system in catheterization laboratory. Cardiovasc Interv Ther. 2013 Jul; 28(3): 235-41. http://dx.doi.org/10.1007/s12928-013-0161-4

[2] Nakao F. Instant Stent-Accentuated 3-Dimensional Optical Coherence Tomography of a Bifurcation Lesion Treated With Reverse Minimum Overlapping Culotte Stenting. JACC Cardiovasc Interv. 2014 Sep; 7(9): e121-2. http://dx.doi.org/10.1016/j.jcin.2014.01.176

[3] Okamura T, Onuma Y, Yamada J, Iqbal J, Tateishi H, Nao T, et al. 3D optical coherence tomography: new insights into the process of optimal rewiring of side branches during bifurcational stenting. EuroIntervention. 2014 Feb 17. pii: 20130514-02. Epub ahead of print.

[4] Alegría-Barrero E, Foin N, Chan PH, Syrseloudis D, Lindsay AC, Dimopolous K, et al. Optical coherence tomography for guidance of distal cell recrossing in bifurcation stenting: choosing the right cell matters. EuroIntervention. 2012 Jun 20; 8(2): 205-13. http://dx.doi.org/10.4244/EIJV8I2A34

[5] Attizzani GF, Capodanno D, Ohno Y, Tamburino C. Mechanisms, pathophysiology, and clinical aspects of incomplete stent apposition. J Am Coll Cardiol. 2014 Apr 15; 63(14): 1355-67. http://dx.doi.org/10.1016/j.jacc.2014.01.019

[6] Ormiston JA, Webber B, Webster MW. Stent longitudinal integrity bench insights into a clinical problem. JACC Cardiovasc Interv. 2011 Dec; 4(12): 1310-7. http://dx.doi.org/10.1016/j.jcin.2011.11.002 